\title{
Tetramethylpyrazine-mediated suppression of C6 gliomas involves inhibition of chemokine receptor CXCR4 expression
}

\author{
KEMING YU $^{1 *}$, ZHAO CHEN $^{1 *}$, XUEKE PAN $^{1}$, YING YANG $^{1}$, SIJIA TIAN ${ }^{1}$, \\ JING ZHANG $^{1}$, JIAN GE ${ }^{1}$, BALA AMBATI ${ }^{2}$ and JING ZHUANG ${ }^{1}$ \\ ${ }^{1}$ State Key Laboratory of Ophthalmology, Sun Yat-sen University, Guangzhou 510060, P.R. China; \\ ${ }^{2}$ Department of Ophthalmology, Moran Eye Center, University of Utah, UT 84132, USA
}

Received April 25, 2012; Accepted May 18, 2012

DOI: $10.3892 /$ or.2012.1866

\begin{abstract}
Tetramethylpyrazine (TMP) is the major component extracted from the Chinese herb Chuanxiong. Increasing numbers of studies have indicated that tetramethylpyrazine hydrochloride (TMPH) has anticancer effects. However, the molecular mechanisms underlying the actions of TMPH have not been fully elucidated. In this study, using real-time RT-PCR and western blot techniques, we demonstrate that TMPH significantly downregulates the expression of the chemokine receptor CXCR4 in C6 glioma cells. Consistent with a role for CXCR4 in cancer development, TMPH inhibits the migration, proliferation and colony formation of $\mathrm{C} 6$ glioma cells in vitro more effectively than the CXCR4 antagonist AMD3100. Interestingly, TMPH does not affect the cell cycle when the cells are grown to 50-80\% confluency but induces S-phase arrest at $100 \%$ confluency, as indicated by a significant reduction in the G1 and G2 populations. These findings were also confirmed in vivo. Rats were implanted with C6 glioma cells and treated with $100 \mathrm{mg} / \mathrm{kg}$ TMPH for 20 days. Our data show that tumour growth was significantly inhibited in rats treated with TMPH $\left(4.14 \pm 2.81 \mathrm{~mm}^{3}\right)$ compared with tumour growth in control rats $\left(55.9 \pm 14.12 \mathrm{~mm}^{3}\right)$. Microcirculation in the implants was sparser in the TMPH-treated rats than that in the control rats, as measured by FITC-dextran staining. Consistent with the in vitro results, TMPH significantly downregulated the expression of CXCR4 in C6 glioma implantation compared with the control. This study provides new insights into the mechanisms of the TMPH anticancer effects.
\end{abstract}

Correspondence to: Dr Jing Zhuang, State Key Laboratory of Ophthalmology, Zhongshan Ophthalmic Center, Sun Yat-sen University, 54 S. Xianlie Road, Guangzhou 510060, P.R. China

E-mail: zhuang@mail.sysu.edu.cn

Dr Bala Ambati, Department of Ophthalmology, Moran Eye Center, University of Utah, Salt Lake City, UT 84132, USA

E-mail: bati@gmail.com

*Contributed equally

Key words: tetramethylpyrazine, glioma, chemokine receptor CXCR4

\section{Introduction}

Chuanxiong (Ligusticum wallichi Franchat) has been commonly used in Chinese traditional medicine for $>2,000$ years. This medicine's bioactive component, tetramethylpyrazine (TMP), was identified and extracted from Chuanxiong in 1973 (1). There have been an overwhelming number of applications of tetramethylpyrazine hydrochloride (TMPH) as a highly efficient clinical treatment for ischemic neurovascular diseases with only mild side effects (2-4). Moreover, many studies have characterised the effects of TMP on tumours as inhibiting the viability and attenuating the metastasis of cancer cells $(5,6)$. Clinical evidence has confirmed that combining TMPH or Chuanxiong with other treatments can significantly attenuate multidrug resistance (MDR) of chemotherapy and increase the sensitivity of cancer cells to radiation in such cancers as nasopharyngeal, lung, breast, renal and ovarian cancer (7-9). Wang et al also confirmed by glioma-neuronal co-culturing that TMP could inhibit the viability of glioma cells while protecting hippocampal neurons and TMP could promote the regression of malignant gliomas in vivo (10). Therefore, TMP may be a potential therapeutic agent for the treatment of gliomas.

Many previous studies have demonstrated that TMP can reduce blood viscosity, manage coagulation and improve the microcirculation around ischaemic tissue $(11,12)$. Therefore, most research groups hypothesise that TMP may increase the radiation and chemotherapy sensitivity of patients by improving the microcirculation around tumour tissue, thereby reducing the side effects on the organs at risk $(7,13)$. Thus, it seems logical to suggest that a correlation exists between the tumour-inhibition and neural-protection bioactivities of TMP. However, the exact molecular mechanisms underlying the actions of TMP have not been fully elucidated.

CXCR4 is a G-protein-coupled receptor with 7 transmembrane-spanning domains that is expressed in various endothelial and tumour cells. Previous studies have demonstrated that CXCR4 plays an important role in all three fundamental aspects of cancer: proliferation, migration, invasion and metastases (13-17). Zhou et al confirmed that CXCR4 was a major chemokine receptor in glioma cells and mediated their survival (13). A recent study reported that the clinically approved drug, AMD3100 (a small molecule inhibitor of SDF-1/CXCR4 
interactions), could significantly prevent the recurrence of glioblastoma in mice after irradiation (18). Currently, CXCR4 has been targeted in the treatment of brain tumours (19-21). In addition, CXCR4 takes different roles when modulating neurotransmission, neurotoxicity and neurological interactions in the mature central nervous system (CNS) $(22,23)$. Abnormal activation of CXCR4 occurs in the pathogenesis of CNS disorders such as stroke, ischemia and multiple sclerosis. Therefore, to determine if TMP regulates CXCR4 expression in glioma, this study was undertaken to test the clinical-grade product, TMPH, both in vitro and in vivo.

\section{Materials and methods}

Cell culture. C6 rat glioma cells were obtained from the ATCC Collection (Manassas, VA) and maintained in DMEM (Invitrogen, USA) supplemented with $10 \%$ FBS plus $100 \mathrm{U} / \mathrm{ml}$ penicillin and $100 \mathrm{mg} / \mathrm{ml}$ streptomycin in a humidified atmosphere of $5 \% \mathrm{CO}_{2}$ at $37^{\circ} \mathrm{C}$. TMPH was purchased from Harbin Medisan Pharmaceutical Co. (China), AMD3100 was purchased from Sigma (USA), and both were dissolved in normal saline to appropriate concentrations. Normal saline was applied as the control in all experiments.

Real-time RT-PCR analysis. Total RNA was isolated with TRIzol Reagent (Invitrogen). Total RNA $(1 \mu \mathrm{g})$ was subjected to reverse transcription using the SYBR PrimeScript ${ }^{\mathrm{TM}}$ RT-PCR kit (Takara, China) following the manufacturer's protocol. Real-time PCR was employed to measure CXCR4, SDF-1 and VEGF expression using the SYBR Green system (Takara). The following primer pairs were used: for CXCR4, 5'-cttatcctgcctggtattgtc-3' and 5'-caatgtagtaaggcagccaac-3'; for SDF-1, 5'-gtcagcctgagctacagatgc-3' and 5'-ttgtttaaggctttgtccaggt-3'; for VEGF, 5'-attgagaccctggtggacatct-3' and 5'-tctctcctatgtgctggctttg-3'; and for $\beta$-actin, 5'-caccacaccttctacaatgag-3' and 5'-tagcacagcctggatagcaac-3'. The quantity of target gene mRNA relative to the internal control gene, $\beta$-actin, was calculated using the $\Delta \mathrm{CT}$ method, as follows: the relative expression $=2^{-\Delta \mathrm{CT}}$, $\Delta \mathrm{CT}=\mathrm{C}_{\mathrm{T}}$ (target gene) $-\mathrm{C}_{\mathrm{T}}(\beta$-actin). The data were analysed in triplicate.

Western blot analyses. Cells were lysed with radio-immunoprecipitation assay buffer. Whole-cell lysates were separated by sodium dodecyl sulphate/polyacrylamide electrophoresis and transferred after $1 \mathrm{~h}$ to a nitrocellulose polyvinylidene fluoride membrane. CXCR4 was detected using primary antibodies against CXCR4 (1:100; Santa Cruz, USA) and a horseradish peroxidase-conjugated goat anti-rabbit secondary antibody (1:500; Santa Cruz). $\beta$-actin served as a loading control. Protein bands were detected using an enhanced chemiluminescence detection system (Santa Cruz, USA).

Cell migration assay. The wound healing assay was performed to quantify the rate of glioma cell migration. C6 glioma cells $\left(1 \times 10^{6}\right)$ were seeded in a $60-\mathrm{mm}$ dish to create a confluent monolayer. After treatment with $200 \mu \mathrm{M}$ TMPH, $10 \mu \mathrm{g} / \mathrm{ml}$ AMD3100 or the control vehicle for $48 \mathrm{~h}$, a wound was created by manually scraping the cell monolayer with a p200-pipette tip. The initial wound quantification was performed on images collected $1 \mathrm{~h}$ after wounding when the wound size had stabilised. Additional images were collected randomly from the wound areas at 6 and $12 \mathrm{~h}$ after wounding.

Cell cycle assay. After treatment with $200 \mu \mathrm{M}$ TMPH, $10 \mu \mathrm{g} /$ $\mathrm{ml}$ AMD3100 or the control vehicle for $48 \mathrm{~h}$, the cells were harvested, fixed with $75 \%$ ice-cold ethanol in PBS and stored at $4^{\circ} \mathrm{C}$. Before analysis, the cells were washed twice with PBS and were incubated for $30 \mathrm{~min}$ in a propidium iodide staining solution $(0.05 \mathrm{mg} / \mathrm{ml}$ propidium iodide, $1 \mathrm{mM}$ EDTA, $0.1 \%$ Triton-X-100 ${ }^{\mathrm{TM}}$ and $1 \mathrm{mg} / \mathrm{ml}$ ribonuclease A) (Sigma-Aldrich, USA). The staining fluorescence intensity was measured by BD FACSort $^{\mathrm{TM}}$ (BD Biosciences, USA) and used to determine the $\mathrm{G} 2 / \mathrm{M}$ ratio.

Viability assays by MTT. C6 glioma cells $\left(5 \times 10^{3}\right)$ were seeded in a 96-well plate in $100 \mu \mathrm{l}$ of medium 1 day prior to addition of $200 \mu \mathrm{M}$ TMPH, $10 \mu \mathrm{g} / \mathrm{ml}$ AMD3100 or the control vehicle. Cell viability was determined by the 3-(4,5-dimethylthiazol2-yl)-2,5-diphenylte tetrazolium bromide (MTT) assay using the optical density ratio of a treated culture over an untreated control.

Colony formation assay. C6 glioma cells $(1,000)$ were seeded in 6-well culture plates in triplicate. After 10 days growth with $200 \mu \mathrm{M}$ TMPH, $10 \mu \mathrm{g} / \mathrm{ml}$ AMD3100 or the control vehicle, the cells were stained with Giemsa for $20 \mathrm{~min}$. The colony formation rate was calculated using Image Pro Plus software (Media Cybernetics) (24).

Tumour implantation. Thirty adult SD rats, each weighing 250-300 g, were provided by the Laboratory Animal Centre of Southern Medical University (Guangzhou, China). The rats were reared in the laboratory animal centre of the Zhongshan Ophthalmic Centre. All experimental procedures were approved by The Ethics Committee of Zhongshan Ophthalmic Centre. Glioma cells $\left(\sim 10^{6}\right)$ were injected into the frontal lobe of the right cerebral hemisphere of the SD rats using a stereotaxic apparatus. Coordinates were set according to the atlas of Paxinos and Watson (25). Seven days after tumour implantation, the SD rats were randomised (15 rats for each group) to a treatment group receiving daily i.p. injections of TMPH at a dose of $100 \mathrm{mg} / \mathrm{kg}$ in $500 \mu \mathrm{l}$ for 20 days or to a control groups receiving i.p. injections of the control vehicle.

Blood vessel staining. To assess tumour angiogenesis, the rats were anesthetised, and $0.1 \mathrm{ml}$ of FITC-dextran solution (50 mg/ml in PBS; Sigma-Aldrich, USA) was administered retro-orbitally. Within 5 min after the injection, animals were sacrificed by cervical dislocation to prevent the FITC-dextran from diffusing distantly from the perfused vessels. Next, the brains were rapidly removed from the cranial cavity and fixed with $4 \%$ paraformaldehyde at $4^{\circ} \mathrm{C}$ for $24 \mathrm{~h}$. Coronal sections (30 $\mu \mathrm{m})$ were cut and observed under a fluorescence microscope. The tumour angiogenesis was analysed using Image Pro Plus software (24).

Statistical analyses. All experiments in vitro were carried out in triplicate. Data are expressed as means \pm SE. The differences between mean values were evaluated with a two-tailed Student's t-test (for 2 groups) and the analysis of variance (ANOVA, for 
A

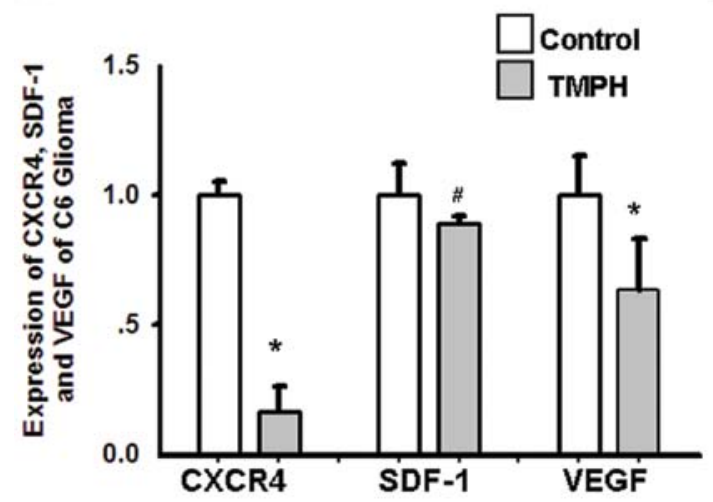

B
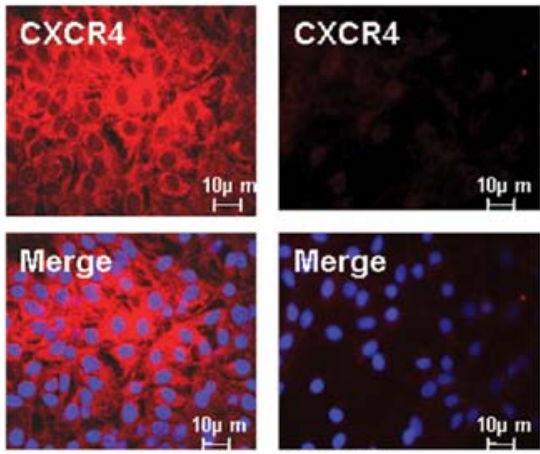

Control

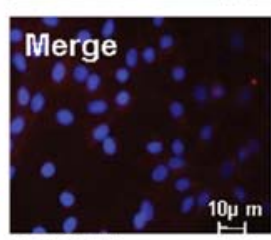

TMPH
C
D

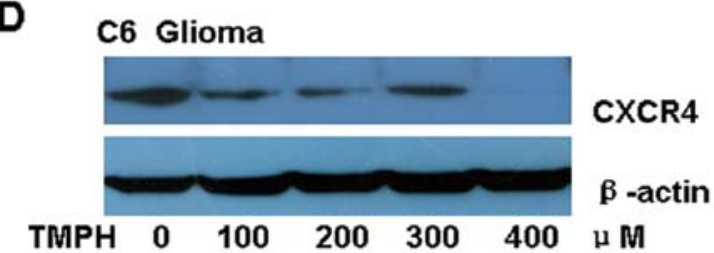

Figure 1. CXCR4 expression is suppressed by $200 \mu \mathrm{M}$ of TMPH. The experiments below were performed $48 \mathrm{~h}$ after treatment with TMPH or the control vehicle: (A) CXCR4, SDF-1 and VEGF expression in C6 glioma cells, as determined by RT-PCR. Error bars, mean \pm SD ( $=3$; ${ }^{*}$ p $<0.001$; ${ }^{\#} \mathrm{p}<0.005$ ). (B) Immunocytofluorescence indicates that expression of CXCR4 in C6 glioma cells (red) is reduced after TMPH treatment (right) compared with the control. (C) Western blotting indicates that TMPH treatment downregulated expression of CXCR4 in C6 glioma cells. (D) Western blotting indicates that TMPH treatment downregulates the expression of CXCR4 in C6 glioma cells in a dose-dependent manner.

$>2$ groups). All calculations and statistical tests were performed using Excel 2003 (Microsoft) or SPSS 11.5 (SPSS). A p $<0.05$ was considered to be significant for all analyses.

\section{Results}

TMPH treatment reduces CXCR4 expression in C6 glioma cells. Considering that CXCR4, stromal cell-derived factor-1 (SDF-1) and VEGF play important roles in cell migration and proliferation, we first measured the expression of CXCR4, SDF-1 and VEGF in C6 glioma cells treated with $100 \mu \mathrm{M}$ TMPH. Fortyeight hours after treatment, total RNA was extracted for analysis by real-time RT-PCR. As shown in Fig. 1A, the expression of CXCR4 and VEGF in C6 glioma cells is down-regulated after TMPH treatment; the decrease in CXCR4 expression is 3.94-fold greater than the decrease in VEGF expression. CXCR4 is the only known receptor for SDF-1, and SDF-1 expression is only slightly altered upon TMPH treatment. The analysis of immunofluorescence staining and western blot data further confirms that TMPH significantly inhibits CXCR4 expression in C6 glioma cells (Fig. 1B and C). Moreover, TMPH treatment downregulates the expression of CXCR4 in glioma cells in a dose-dependent manner (Fig. 1D).

TMPH inhibits cell migration and viability. The SDF-1/CXCR4 interaction plays a pivotal role in cell migration. AMD3100 is a specific inhibitor of CXCR4. Therefore, the effects of TMPH $(200 \mu \mathrm{M})$ on cell migration were evaluated using a scratchwound assay, and the results were compared to those from cells treated with $10 \mu \mathrm{g} / \mathrm{ml}$ of AMD3100. As shown in Fig. 2A, TMPH significantly decreases the migration of C6 glioma cells compared to the control. More open spaces are observed at 6 and
$12 \mathrm{~h}$ after treatment with TMPH. Moreover, the inhibition of cell migration by TMPH is more effective than that of AMD3100. Cell viability was measured by staining with MTT. Fig. 2B shows that TMPH inhibits the viability of cultured glioma cells and that this inhibition is much stronger than that caused by AMD3100.

Previous data regarding the effects of AMD3100 on the cell cycle were controversial $(13,26,27)$. To determine the bioactivity of TMPH on cell growth and to compare the bioactivity of TMPH and AMD3100, we analysed the cell cycle profile of C6 glioma cells at different confluencies. As shown in Fig. 2C, at $48 \mathrm{~h}$ after treatment, when the cells are 50-80\% confluent, TMPH does not alter the cell cycle profile of glioma cells. However, when the cells are $100 \%$ confluent, TMPH treatment induces an arrest in $\mathrm{S}$ phase $(51.52 \pm 2.29 \%)$ compared to treatment with AMD3100 $(37.5 \pm 2.63 \%)$ and the control $(32.95 \pm 1.28 \%)$. The rate of cell growth in the G1 $(34.10 \pm 1.53 \%)$ and $\mathrm{G} 2$ phases $(14.03 \pm 1.54 \%)$ is significantly reduced in glioma cells treated with TMPH compared to the control (G1 phase $44.22 \pm 1.70 \%$, G2 phase $22.83 \pm 2.16 \%)$. AMD3100 does not affect $S$ phase $(37.51 \pm 2.62 \%)$, but there are more cells in G1 phase $(57.10 \pm 2.88 \%)(\mathrm{p}<0.001)$ and fewer in $\mathrm{G} 2$ phase $(5.39 \pm 1.51 \%)(\mathrm{p}<0.001)$ (Fig. 2D). These data indicate that TMPH and AMD3100 affect different phases of the cell cycle.

TMPH inhibits colony formation of C6 glioma cells. There is increasing evidence that the capacity of cancer cells to form colonies is critical for the process of tumour development $(28,29)$. To determine the impact of TMP on colony formation, 1,000 C6 glioma cells were seeded into a 6-well plate, and colony formation was recorded after 10 days, at which time colonies are visible. As shown in Fig. 3, TMPH effectively inhibits colony formation of the seeded cells compared with the 
A Control
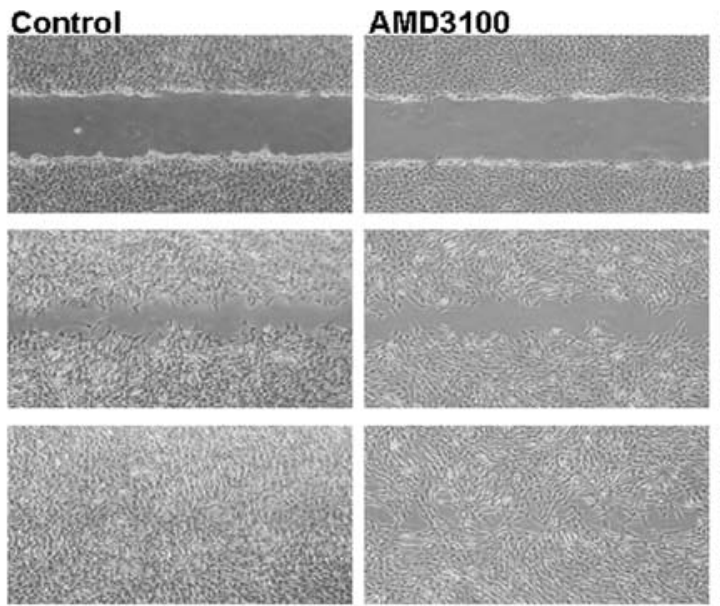

C
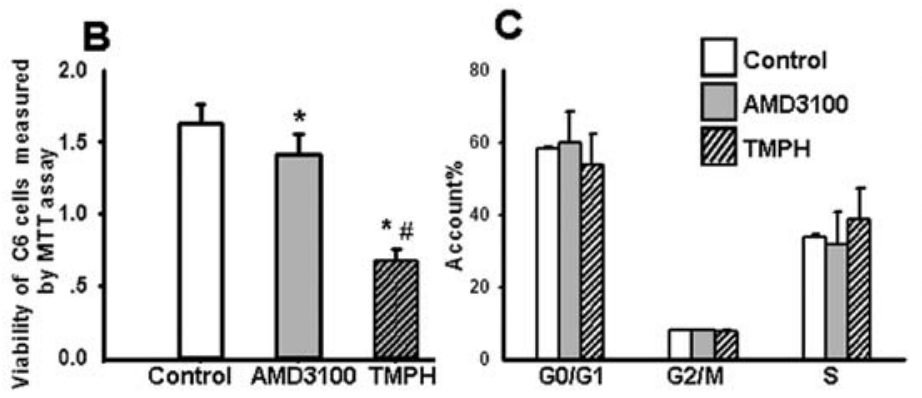

TMPH
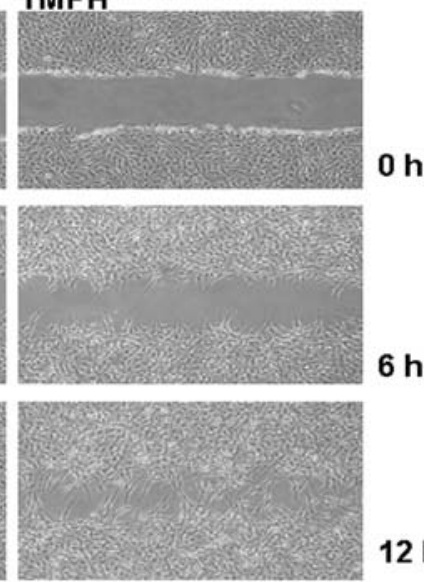

$12 \mathrm{~h}$

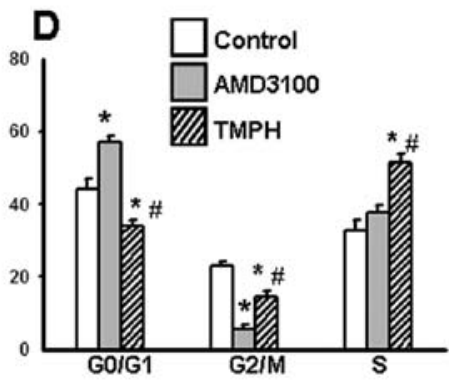

Figure 2. TMPH suppresses cell migration and viability. (A) Scratch-wound assay of C6 glioma cells. The glioma cells were seeded on 6-well plates in the presence of $200 \mu \mathrm{M} \mathrm{TMPH}, 10 \mu \mathrm{g} / \mathrm{ml}$ AMD3100 or the control vehicle for $48 \mathrm{~h}$ and were wounded with a sterile pipette tip to remove cells from two perpendicular linear scratches. Representative phase contrast images show that the wound induces glioma cells migration after $12 \mathrm{~h}$. Original magnification $\mathrm{x} 40$. (B) Growth of glioma cells is significantly inhibited by TMPH and AMD3100 treatment. Error bars, mean \pm SD (n=4). (C) When the cells are 50-80\% confluent, TMPH and AMD3100 treatment does not affect the cell cycle profiles of glioma cells. (D) When the cells are $100 \%$ confluent, they become arrested in $\mathrm{S}$ phase and fail to enter the G2/M and G1 phases after TMPH treatment compared with the control. In contrast, there is no difference in the S phase populations between cells treated with AMD3100 and the control. The cells are arrested in the G1 phase after AMD3100 treatment. "Statistically significant differences between the control, ADM3100 and TMPH; ${ }^{\#}$ statistically significant differences between TMPH and AMD3100 ( $\left.{ }^{*} \mathrm{p}<0.001 ;{ }^{\#} \mathrm{p}<0.001\right)$.

A

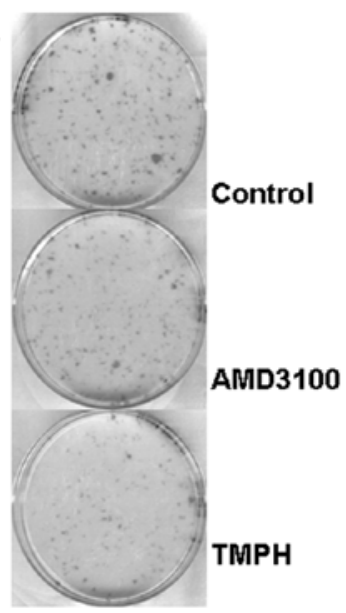

B

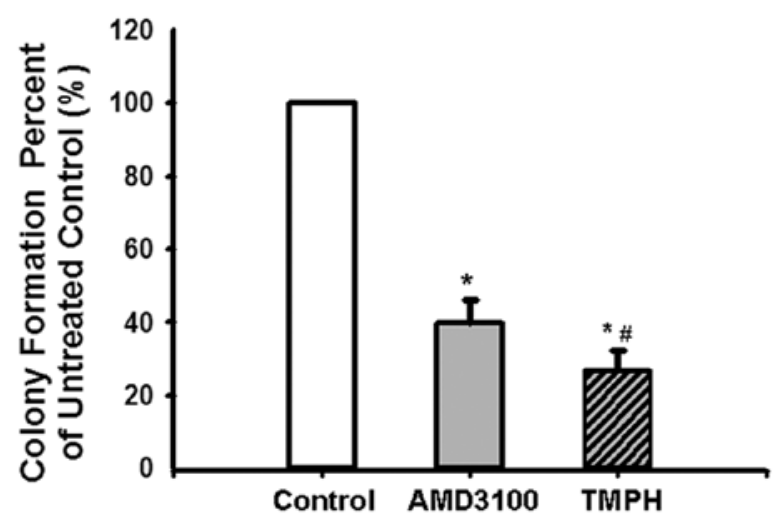

Figure 3. (A) TMPH suppresses glioma cell colony formation. (B) The colony formation rates are presented in histograms. Error bars, mean \pm SD ( $=5$, 3). "Statistically significant differences between TMPH, ADM3100 and the control; " statistically significant differences between TMPH versus AMD3100 (*p<0.001; " $\mathrm{p}<0.001)$.

control. Moreover, the colony formation rate was significantly decreased in glioma cells treated with TMPH $(26.49 \pm 5.65 \%)$ compared with AMD3100 (39.68 $\pm 6.50 \%)$.

TMPH causes malignant glioma regression in vivo. To validate our findings that TMPH-mediated inhibition of glioma cells involves inhibition of CXCR4-dependent migration in vivo, $\sim 10^{6}$ glioma cells were implanted into the frontal lobe of the left cerebral hemisphere of SD rats using a stereotaxic apparatus. Seven days after tumour implantation, the SD rats were randomised to treatment groups receiving i.p. injections of TMPH or normal saline daily for 20 days. Fig. 4A shows that the size 
A

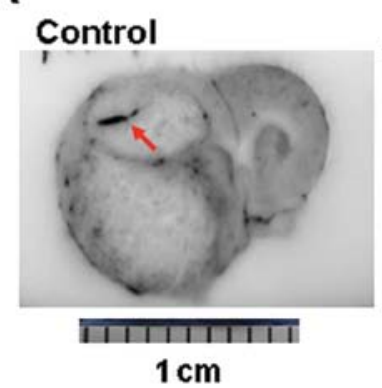

TMPH

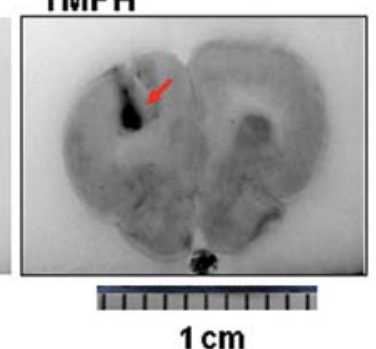

B

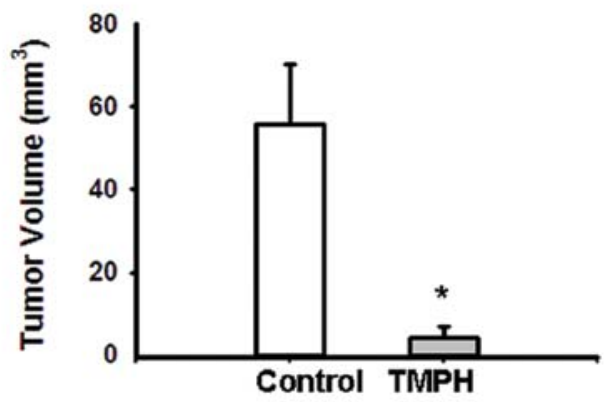

C control

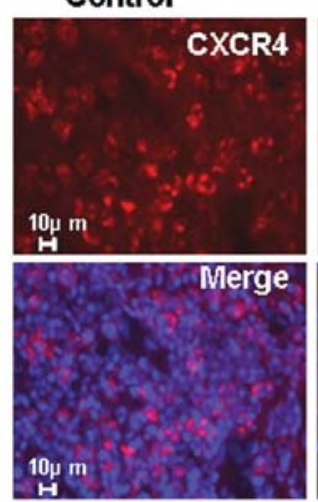

TMPH

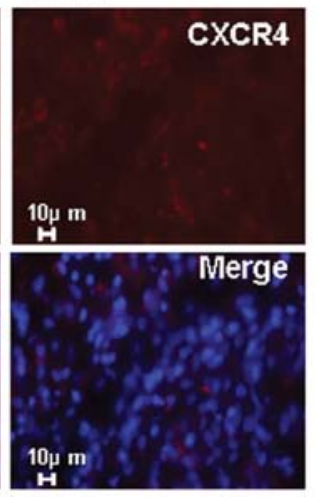

D

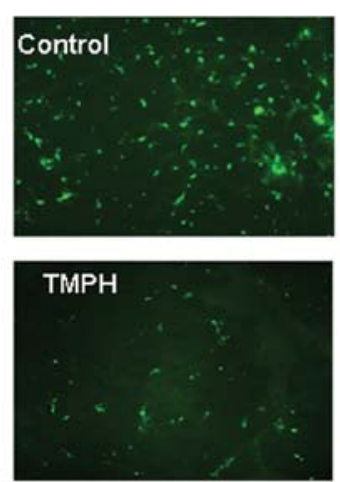

E

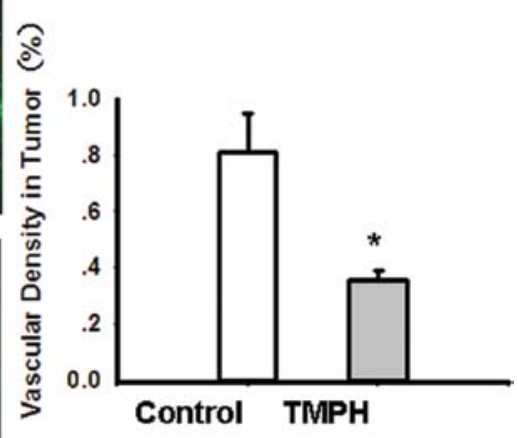

Figure 4. TMPH-induced regression of gliomas in SD rats. (A) Photomicrographs showing tumours from the control group on day 20. A haemorrhage is labelled with a red arrowhead. Original magnification $\mathrm{x} 40$. (B) The average tumour volumes in the control $\left(55.9 \pm 14.12 \mathrm{~mm}^{3}\right)$ and TMPH-treated $\left(4.14 \pm 2.81 \mathrm{~mm}^{3}\right)$ gliomas at 20 days after implantation of glioma cells. Error bars, mean \pm SD ( $\left.n=10 ;{ }^{*} \mathrm{p}<0.001\right)$. (C) Immunocytofluorescence indicates that CXCR4 (red) is reduced in the glioma after TMPH treatment. (D) Photomicrographs showing FITC-dextran (green) perfused microcirculation in the control and TMPH-treated gliomas at day 20 after glioma cell implantation. (E) The bar graph represents the mean and the SE of FITC-dextran (green)-perfused microcirculation within the microscopic fields from 10 experiments $\left(\mathrm{n}=10 ;{ }^{*} \mathrm{p}<0.001\right)$.

of a C6 glioma implantation treated with TMPH is significantly smaller than that of the control. There is obvious haemorrhaging in the C6 glioma implantation after TMPH treatment (red arrowhead). Tumour growth is significantly inhibited in rats treated with $0.8 \mathrm{mg}$ TMPH day-1 $\left(4.14 \pm 2.81 \mathrm{~mm}^{3}\right)$ compared with the growth in control rats $\left(55.9 \pm 14.12 \mathrm{~mm}^{3}\right)(\mathrm{p}<0.001)$ (Fig. 4B). These data are consistent with a previous report on the effects of TMP treatment in a rat model of C6 glioma (10). Consistent with our results from $\mathrm{C} 6$ glioma cells in vitro, TMPH downregulates CXCR4 in glioma implantation compared with the control (Fig. 4C).

To verify the role of CXCR4 signalling during vascular response in vivo, FITC-dextran was injected as a perfusion marker before the tumours were harvested. As shown in Fig. 4D, the microcirculation in the tumour is denser in the control rats than in the TMPH-treated rats. Fig. 4E shows the quantification of microvasculature formation as measured by microscopy $(\mathrm{p}<0.001)$.

\section{Discussion}

In the present study, we demonstrated that the CXCR4/SDF-1 pathway is a novel mechanism underlying TMP-mediated glioma inhibition. Our results demonstrated that TMPH significantly decreased CXCR4 expression from rat C6 glioma cells (Fig. 1A-C). We also found that TMPH treatment downregulates the VEGF expression in C6 glioma cells (Fig. 1A), which has previously been considered to be the main factor in TMP treatment of tumours. However, the decrease in CXCR4 expression induced by TMPH is 3.94-fold greater than the decrease in VEGF expression. Moreover, a recent study demonstrated that the CXCR4/SDF-1 axis was the major cause of ectopic overexpression of VEGF in tumours. The CXCR4/SDF-1 axis regulates VEGF expression through Yin Yang 1 (YY1), which is a positive regulator of VEGF (30). Based on this evidence, we deduce that the downregulation of CXCR4 induced by TMP treatment occurs prior to reducing VEGF expression.

SDF-1/CXCR4 plays a key role in promoting the migration and growth of tumour cells (17). Our study demonstrated that TMPH treatment significantly decreased the migration and viability of C6 glioma cells (Fig. 2A). Moreover, this inhibition by TMPH is more effective than the CXCR4 antagonist, AMD3100.

We analysed the cell cycle profiles of C6 glioma cells after TMPH treatment. We found that TMPH and AMD3100 do not affect the cell cycle when the cells are 50-80\% confluent. However, at $100 \%$ confluency, TMPH induces arrest in S phase, significantly reducing the G1 and G2 populations of C6 glioma cells compared with that of the control. Similarly, AMD3100 also affects the cell cycle when the cells are $100 \%$ confluent. These results are consistent with the previous controversial reports on the effects of AMD3100 $(13,26,27)$ on the cell cycle. TMP and AMD3100 affect cell cycle in a dose-dependent manner in some studies but not in others. Moreover, there is a 
significant difference in cell cycle profiles between the treatment with TMP and AMD3100 at 100\% confluency. The cells treated with AMD3100 were arrested in the G1 phase compared with the control. Therefore, the basis of the fundamental physiological roles of TMPH and AMD3100 in the cell cycle or cell migration may be that TMP directly downregulates CXCR4, while AMD3100 directly blocks the active domain of CXCR4.

We further evaluated the bioactivity of TMPH on C6 glioma cell implantation in vivo. Our data show that tumour growth is significantly inhibited in rats treated with TMPH compared with the control, which is in agreement with previous reports $(10,18)$. Consistent with our in vitro results with C6 glioma cells, the expression of CXCR4 is abolished in C6 glioma cell implantation after TMPH treatment.

Moreover, we also confirmed that TMPH could protect cerebral neurocytes by downregulating the expression of CXCR4 and inhibiting glutamate release from cerebral neurocytes in vitro (unpblished data), which build up a correlation existing between the tumour-inhibition and neural-protection bioactivities of TMP.

In conclusion, we believe we have shown for the first time that TMP treatment results in inhibition of cell migration, thereby blocking the cell cycle and eventually suppressing tumour growth by downregulating CXCR4 expression in glioma cells both in vitro and in vivo. This finding is in contrast to the widely held notion that TMP exerts bioactivity only through improving microcirculation (7,13). Additionally, TMPH could be a potential agent for treatment of malignant brain tumours.

\section{Acknowledgements}

This study was supported was supported by the grant from the National Natural Science Foundation (Project: 30872811).

\section{References}

1. http://www.bjkw.gov.cn/n1143/n1240/n1465/n2261/n240731/ n241840/405420. html.

2. Liu SF, Cai YN, Evans TW, et al: Ligustrazine is a vasodilator of human pulmonary and bronchial arteries. Eur J Pharmacol 191: 345-350, 1990.

3. Shen SY, Fu XD and Fei ZY: Assessment and explorations on the mechanism of neuroprotection of patients in ischemic stroke by traditional Chinese medicine. Chin J Integr Med 11: 237-240, 2005 (In Chinese).

4. Han Z, Wang Q, Gao F, Ren Y and Yang KJ: The clinic evaluation of treatment of Ligustrazine and Radix Astragalus to 36 patients. J Fourth Military Medical University 27: 1207-1209, 2006 (In Chinese)

5. Wang XB, Wang SS, Zhang QF, et al: Inhibition of tetramethylpyrazine on P-gp, MRP2, MRP3 and MRP5 in multidrug resistant human hepatocellular carcinoma cells. Oncol Rep 23: 211-215, 2010.

6. Mei Y, Shi Y, Zuo G, Gong J and Liu C: Study on ligustrazine in reversing multidrug resistance of HepG2/ADM cell in vitro. Zhongguo Zhong Yao Za Zhi 29: 970-973, 2004.

7. Li Z, Shao M, Zhou Y, Wei R and Gong Y: Resistance-related protein in rectal cancer tissue expression and significance of combination chemotherapy with Ligustrazine. Int Med Health Guidance News 2: 142-144, 2010 (In Chinese).

8. Li L, Xu K, Liu Y, Zheng J, Qiu S and Liu C: The clinic evaluation of treatment of percutaneous implantation of radioactive particles with the TMP to 20 patients with malignant cancer by CT-guided. Shaanxi J Trad Chin Med 29: 542-544, 2008.
9. Wu Y, Dai X, Hu P and Chen Z: The progresses of Chinese traditional medicine on brain tumors. J New Chin Med 38: 76-79, 2004.

10. Fu YS, Lin YY, Chou SC, et al: Tetramethylpyrazine inhibits activities of glioma cells and glutamate neuro-excitotoxicity: potential therapeutic application for treatment of gliomas. Neurooncology 10: 139-152, 2008.

11. Li M, Handa S, Ikeda Y and Goto S: Specific inhibiting characteristics of tetramethylpyrazine, one of the active ingredients of the Chinese herbal medicine 'Chuanxiong', on platelet thrombus formation under high shear rates. Thromb Res 104: 15-28, 2001.

12. Liu Y, Ren J, Jiang H, Du S and Zhang Y: The influenced foundation of hemodynamics during recovery of blood stasis rats by gustrazine and angelica parenteral solution. Chin J Med Physics 22: 680-681, 2005.

13. Zhou Y, Larsen PH, Hao C, et al: CXCR4 is a major chemokine receptor on glioma cells and mediates their survival. J Biol Chem 277: 49481-49487, 2002.

14. Wang X and Chen X: Study on the effects of tetramethylpyrazine on tumor cells: survey and prospects. Zhongguo Zhong Yao Za Zhi 28: 295-298, 2003.

15. Muller A, Homey B, Soto H, et al: Involvement of chemokine receptors in breast cancer metastasis. Nature 410: 50-56, 2001.

16. Righi E, Kashiwagi S, Yuan J, et al: CXCL12/CXCR4 blockade induces multimodal antitumor effects that prolong survival in an immunocompetent mouse model of ovarian cancer. Cancer Res 71: 5522-5534, 2011.

17. Ping YF, Yao XH, Jiang JY, et al: The chemokine CXCL12 and its receptor CXCR4 promote glioma stem cell-mediated VEGF production and tumour angiogenesis via PI3K/AKT signaling. J Pathol 224: 344-354, 2011.

18. Kioi M, Vogel H, Schultz G, et al: Inhibition of vasculogenesis, but not angiogenesis, prevents the recurrence of glioblastoma after irradiation in mice. J Clin Invest 120: 694-705, 2010.

19. Terasaki M, Sugita Y, Arakawa F, Okada Y, Ohshima K and Shigemori M: CXCL12/CXCR4 signaling in malignant brain tumors: a potential pharmacological therapeutic target. Brain Tumor Pathol 28: 89-97, 2011.

20. Schulte A, Gunther HS, Phillips HS, Kharbanda S, Soriano RH, Modrusan Z, Zapf S, Westphal M and Lamszus K: A distinct subset of glioma cell lines with stem cell-like properties reflects the transcriptional phenotype of glioblastomas and overexpresses CXCR4 as therapeutic target. Glia 59: 590-602, 2011.

21. Schulte A, Gunther HS, Phillips HS, et al: CXCL12 (SDF1alpha)-CXCR4/CXCR7 pathway inhibition: an emerging sensitizer for anticancer therapies? Clin Cancer Res 17: 2074-2080, 2011.

22. Calì C and Bezzi P: CXCR4-mediated glutamate exocytosis from astrocytes. J Neuroimmunol 224: 13-21, 2010.

23. Cartier L, Hartley O, Dubois-Dauphin M and Krause KH: Chemokine receptors in the central nervous system: role in brain inflammation and neurodegenerative diseases. Brain Res Brain Res Rev 48: 16-42, 2005.

24. Zhou Q, Kiosses WB, Liu J and Schimmel P: Tumor endothelial cell tube formation model for determining anti-angiogenic activity of a tRNA synthetase cytokine. Methods 44: 190-195, 2008.

25. Paxinos $G$ and Watson $C$ (eds): The rat brain in stereotaxic coordinates. 2nd edition. Academic Press, San Diego, CA, pp567-572, 1986.

26. Redjal N, Chan JA, Segal RA and Kung AL: CXCR4 inhibition synergizes with cytotoxic chemotherapy in gliomas. Clin Cancer Res 12: 6765-6771, 2006.

27. Rubin JB, Kung A, Klein RS, et al: A small-molecule antagonist of CXCR4 inhibits intracranial growth of primary brain tumors, Proc Natl Acad Sci USA 100: 13513-13518, 2003.

28. Egeblad M and Werb Z: New functions for the matrix metalloproteinases in cancer progression. Nat Rev Cancer 2: 161-174, 2002.

29. Shoemaker RH, Wolpert-DeFilippes MK, Kern DH, et al: Application of a human tumor colony-forming assay to new drug screening. Cancer Res 45: 2145-2153, 1985.

30. Nigris Fd, Crudele V, Giovane A, et al: CXCR4/YY1 inhibition impairs VEGF network and angiogenesis during malignancy. Proc Natl Acad Sci USA 107: 14484-14489, 2010. 